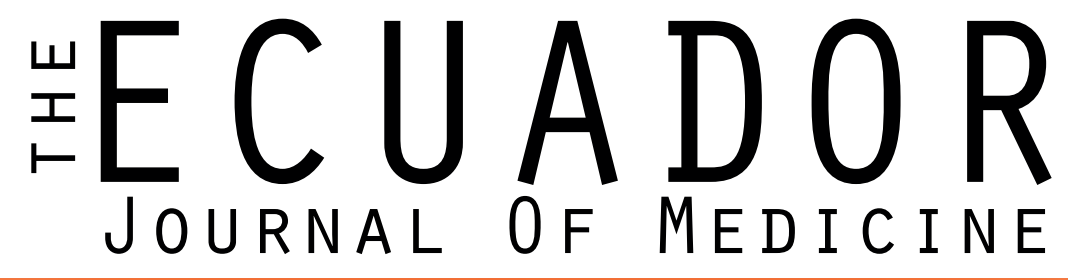

\title{
AUTOMEDICACIÓN Y CONSUMO DE ESTIMULANTES EN ESTUDIANTES DE MEDICINA Y OTRAS FACULTADES EN LA UNIVERSIDAD DEL AZUAY 2013 - 2014
}

\section{ARTÍCULO ORIGINAL}

Natalia Pacurucu

Merchán, $\mathrm{MD}_{\mathrm{a}}$

María Verónica

Muñoz, $\mathrm{MD}_{\mathrm{b}}$

${ }^{a}$ Médico general

${ }^{\mathrm{b}}$ Médico general, egresada de posgrado de ginecologia y obstetricia de la Universidad de Cuenca.

Año realizado el caso

2015

ORCID:0000-0002-4370-2139 0000-0003-1173-191X ISSN: $2737-6486$

\section{RESUMEN}

Objetivo: Comparar la automedicación y consumo de bebidas estimulantes en estudiantes de medicina y otras facultades de la Universidad del Azuay durante abril de 2013 a mayo de 2014.

Materiales y métodos: Se realizó un estudio trasversal en una muestra de 502 estudiantes, 251 de medicina (población) y 251 de otras facultades las cuales fueron aleatorizadas de una población de 5800 estudiantes de manera proporcional al número de integrantes de cada facultad. Los datos se obtuvieron mediante un cuestionario auto administrado, se analizaron en medidas de tendencia central, tablas de frecuencia y las comparaciones se realizaron con la prueba del Chi cuadrado de independencia, tomándose como significativo el valor de p menor 0.05 .

Resultados: Del grupo de estudio (502 estudiantes), 53.5\% fueron mujeres, el promedio de edad fue de 21 años (DS $\pm 2,83$ ), 94.1\% fueron solteros. El grupo de medicina y otras facultades tenían promedio de edad de 21 años (p 0,081). El porcentaje de mujeres fue similar en ambos grupos $(56,6 \%$ y $50,6 \%$, p 0,179). El $96,4 \%$ y $98 \%$ de estudiantes de medicina y otras facultades respectivamente, consumen algún tipo de sustancia estimulante (p 0,278). El consumo con drogas estimulantes (modafinilo, cafeína, ritalina) fue $52,2 \%$ en medicina y $37,5 \%$ en otras facultades ( $p<0,001)$. El consumo de bebidas estimulantes fue de 92 a $94 \%$ (p 0,380). En medicina el 80\% presentaron efectos adversos, y en otras facultades $73 \%$ (p 0,027). Conclusión: La automedicación y el consumo de bebidas estimulantes en estudiantes de la universidad del Azuay son similares a otros estudios. Los estudiantes de medicina consumen más estimulantes que las otras facultades.

Palabras clave: Automedicación, medicamentos estimulantes, bebidas estimulantes.

\section{ABSTRACT}

Objective: Compare self-medication and consumption of energy drinks in students from medical school and other faculties of Universidad del Azuay.

Materials and methods: A cross-sectional study on a simple of 502 students was conducted, 251 were from medical school (population) and 251 from other faculties; samples randomized in a population of 5800 students in a proportion to the number of members of each faculty. The data were obtained by means of a self-administered quetionnaire, and were analyzed in measures of central tendency, and frecuency tables. The comparisons were done using Chi-square test of Independence, taking as significant p value less 0.05 .

Results: From the study group (502 students), 53.5\% were women; the average age was 21 (DS $\pm 2,83$ ), $94.1 \%$ were single. The group of medical students and other faculties were 21 as average age (p 0,081). The percentage of women was similar in both groups $(56,6 \%$ y $50,6 \%, p 0,179) .96 .4 \%$ and $98 \%$ of medical students and other faculties respectively, cinsume some type of stimulant ( 0,278$)$. The stimulant drug consumption (modafinil, caffeine, ritalin) was $52.2 \%$ among medical students and $37.5 \%$ in other faculties ( $p<0,001$ ). The consumption of stimulating beverages was 92 a $94 \%$ ( 0 0,380). In medical students, $80 \%$ experienced adverse effects, and 73\% ( $p$ 0,027) in other faculties. Conclusion: Data regarding self-medication and consumption of stimulants in students from Universidad del Azuay is similar to other studies. Medical students consume more stimulating than the other faculties.

Keywords: self-medication, stimulant drugs, stimulating beverages. 


\section{THE ECUADOR JOURNAL OF MEDICINE}

\section{IN TRODUCC I ÓN}

a automedicación es el uso de fármacos, sin receta, por iniciativa propia de las personas. Este es un problema de salud pública universal, debido a los innecesarios riesgos que implica pues incrementa la posibilidad de reacciones adversas; $\mathrm{O}$, incluso, potencia la acción de los ya mencionados $(1,2)$. Esta conducta puede ser, en parte, la respuesta más común ante enfermedades; e, inclusive, en relación a las bebidas estimulantes, dada su influencia social y comercial, sin que se considere, de parte de quienes las consumen, los efectos adversos que estos provocan, como gastritis, diarrea, náusea, vómito, insomnio, ansiedad, cefalea, alteración en el ritmo cardiaco, entre otros. No existen estudios recientes a nivel nacional que permitan conocer la prevalencia de este problema, razón por la cual se decide realizar un estudio mediante encuestas, una investigación de bajo costo, de la cual se pueda obtener información de una población susceptible como los estudiantes universitarios de ciencias de la salud comparados con estudiantes de otras carreras
(3), ya que los primeros mencionados tienen mayor acceso y conocimiento del uso de fármacos por el medio en el que se desenvuelven $(4,5)$.

A nivel nacional se dispone de escasa información sobre el tema por lo cual este estudio aporta datos locales en una población universitaria, no implicó riesgos biológicos ni psicológicos para los estudiantes que participaron en esta investigación y los resultados servirán para la detección temprana del comportamiento de riesgo en el ámbito de la automedicación con estimulantes para tomar medidas de prevención, además de abrir paso a futuras investigaciones. La percepción es que los estudiantes de medicina se automedican y consumen mayor cantidad de bebidas estimulantes, probablemente por la exigencia académica a la que están sometidos. Por lo mencionado se plantea que la automedicación y consumo de bebidas estimulantes es diferente entre los estudiantes de medicina y las otras facultades. 


\section{THE ECUADOR JOURNAL OF MEDICINE}

\section{MATERIAL Y MÉTODOS}

Se realizó un estudio descriptivo transversal, a 502 estudiantes de las facultades de la Universidad del Azuay desde abril de 2013 a mayo de 2014, en la ciudad de Cuenca.

Se tomó al universo de medicina correspondiente a 251 estudiantes y de igual tamaño correspondiente a otras facultades, de manera aleatoria, de un total de 5800 alumnos, matriculados en el período mencionado. De tal manera 25 fueron de ciencias jurídicas, 51 de filosofía, 46 de diseño, 66 de administración y 63 de ciencia y tecnología.
Para el análisis estadístico se utilizó el software SPSS versión 15; se estableció variables socio-demográficas (edad, sexo, estado civil) y características de la automedicación como consumo de sustancias estimulantes y efectos adversos, que fueron recolectados mediante un cuestionario auto administrado (6). Los datos se analizaron en medidas de tendencia central, tablas de frecuencia y las comparaciones se realizaron con la prueba del Chi cuadrado de independencia, tomándose como significativo el valor de p menor 0.05 .

\section{RESULTADOS}

Se estudió a 502 estudiantes de la Universidad del Azuay durante el período abril 2013 a mayo 2014, de los cuales 251 son de medicina y 251 de otras facultades. El promedio de edad del grupo de estudio fue de 21 años (DS \pm 2,83 ), las edades oscilaron entre 17 y 34 años. El $53,5 \%$ fueron mujeres y $46.5 \%$ nombres, $94.2 \%$ solteros, $4,7 \%$ casados, $1.1 \%$ unión libre (Tabla 1). El 97,2\% consumen algún tipo de sustancia estimulante (droga o bebida).

Tanto en el grupo de medicina como en otras facultades la edad media fue de 21 años $(p 0,081)$. El porcentaje de mujeres en medicina fue $56,6 \%$ y en otras facultades de 50,6\% (p 0,179). Se encontró que el $96,4 \%$ y $98 \%$ de estudiantes de medicina y otras facultades respectivamente, consumen algún tipo de sustancia estimulante (p $0,278)$. El consumo con drogas estimu- lantes como modafinilo, cafeína y ritalina fue $52,2 \%$ en medicina y $37,5 \%$ en otras facultades ( $p<0,001$ ) (tabla 4 ). El consumo de bebidas estimulantes fue elevado en ambos grupos de 92 en medicina y $94 \%$ en otras facultades (p 0,380); las de mayor consumo en el grupo de medicina fueron las bebidas energizantes (V220, RedBull, Monster) seguido de café y té. En otras facultades el orden de frecuencia de consumo fue café, bebidas energizantes y té (tabla 3). El $80 \%$ de estudiantes de medicina presentaron efectos adversos entre los más comunes fueron neurológicos (cefalea, insomnio, mareo, vértigo, euforia, confusión mental) y cardiológicos (palpitaciones y taquicardia) (Tabla 5). El grupo de otras facultades presentó $73 \%$ de efectos adversos predominando los neurológicos (Tabla 2). 


\section{TABLA 1. \\ THE ECUADOR JOURNAL OF MEDICINE}

Características generales del grupo de ESTudio, Universidad del AZUAY $2013-2014$

\begin{tabular}{|l|c|c|}
\hline Variables & Frecuencia (n=520) & $\%$ \\
\hline Sexo & \multicolumn{2}{|l|}{} \\
\hline Masculino & 233 & 46,5 \\
\hline Femenino & 269 & 53,5 \\
\hline Grupo etario & \multicolumn{2}{|l|}{} \\
\hline$<20$ años & 158 & 31,5 \\
\hline $20-25$ & 317 & 63,1 \\
\hline$>25$ años & 27 & 5,4 \\
\hline Estado Civil & & \\
\hline Solteros & 473 & 94,2 \\
\hline Casados & 24 & 4,7 \\
\hline Unión Libre & 5 & 1,1 \\
& & \\
\hline
\end{tabular}

Fuente: formularios de recolección de datos

Elaborado por las autoras

TABLA 2,

COMPARACIÓN DE LA AUTOMEDICACIÓN Y CONSUMO DE BEBIDAS ESTIMULANTES EN ESTUdiANTES DE MEDICINA Y OTRAS FACULTADES DE LA UNIVERSIDAD DEL AZUAY 2013 - 2014

\begin{tabular}{|c|c|c|c|}
\hline Variables & $\begin{array}{l}\text { Medicina } \\
(\mathrm{n}=251)\end{array}$ & $\begin{array}{c}\text { Otras } \\
\text { Facultades } \\
(\mathrm{n}=251)\end{array}$ & $P$ \\
\hline \multicolumn{4}{|c|}{ Automedicación 1} \\
\hline SI & $78.1 \%(\mathrm{n}=196)$ & $68 \%(n=170)$ & \multirow[t]{2}{*}{0,009} \\
\hline No & $21.9 \%(n=55)$ & $32 \%(n=81)$ & \\
\hline \multicolumn{4}{|c|}{$\begin{array}{l}\text { Fármacos Estimulantes } \\
\text { (Modafinilo, Ritalina, cafeina) }\end{array}$} \\
\hline SI & $52.2 \%(n=131)$ & $37.5 \%(n=94)$ & \multirow[t]{2}{*}{$<0,001$} \\
\hline No & $47.8 \%(n=157)$ & $62.5 \%(n=157)$ & \\
\hline \multicolumn{4}{|c|}{ Consumo Bebidas } \\
\hline SI & $92 \%(n=231)$ & $94 \%(n=235)$ & \multirow[t]{2}{*}{0,380} \\
\hline NO & $8 \%(n=20)$ & $6 \%(n=16)$ & \\
\hline \multicolumn{4}{|c|}{ Efectos Adversos } \\
\hline SI & $80 \%(n=201)$ & $73 \%(n=183)$ & \multirow[t]{2}{*}{0,027} \\
\hline No & $20 \%(n=50)$ & $27 \%(n=68)$ & \\
\hline
\end{tabular}

incluye: fármacos estimulantes, relajantes musculares, ansiolíticos, antidepresivos, anfetaminas y cocaína.

Fuente: formularios de recolección de datos.

Elaborado por las autoras 
TABLA 3.

THE ECUADOR JOURNAL OF MEDICINE

Consumo de bebidas estimulantes del gRupo de Estudio, Universidad DEL AZUAY $2013-2014$

\begin{tabular}{|c|c|c|}
\hline $\begin{array}{c}\text { Bebidas } \\
\text { estimulantes }\end{array}$ & Medicina & $\begin{array}{c}\text { Otras } \\
\text { facultades }\end{array}$ \\
\hline $\begin{array}{c}\text { Bebidas } \\
\text { energizantes }\end{array}$ & $84.06 \%$ & $78.5 \%$ \\
\hline Café & $74.5 \%$ & $80.1 \%$ \\
\hline Té & $56.6 \%$ & $66.5 \%$ \\
\hline
\end{tabular}

Fuente: formularios de recolección de datos

Elaborado por las autoras

TABLA 4 ,

CONSUMO DE SUSTANCIAS ESTIMULANTES DEL GRUPO DE ESTUDiO, UNIVERIDAD DEL AZUAY $2013-2014$

\begin{tabular}{|c|c|c|}
\hline $\begin{array}{c}\text { Sustancias } \\
\text { automedicadas }\end{array}$ & Medicina & $\begin{array}{c}\text { Otras } \\
\text { facultades }\end{array}$ \\
\hline $\begin{array}{c}\text { Medicamentos } \\
\text { Estimulantes }\end{array}$ & $52.2 \%$ & $37.5 \%$ \\
\hline $\begin{array}{c}\text { Ansioliticos } \\
\text { Antidepresivos }\end{array}$ & $6 \%$ & $17.53 \%$ \\
\hline $\begin{array}{c}\text { Relajantes } \\
\text { Musculares }\end{array}$ & $29.5 \%$ & $13.2 \%$ \\
\hline $\begin{array}{c}\text { Cocaina } \\
\text { Anfetaminas }\end{array}$ & $1.2 \%$ & $41.8 \%$ \\
\hline
\end{tabular}

Fuente: formularios de recolección de datos

Elaborado por las autoras

TABLA 5 ,

Efectos adversos del grupo de estudio, Universidad del azuay $2013-2014$

\begin{tabular}{|c|c|c|}
\hline Efectos adversos & Medicina & $\begin{array}{c}\text { Otras } \\
\text { facultades }\end{array}$ \\
\hline Gastrointestinales & $32.5 \%$ & $26.8 \%$ \\
\hline Cardiológicos & $66.6 \%$ & $68.75 \%$ \\
\hline Neurológicos & $86 \%$ & $30.1 \%$ \\
\hline Generales & $15.7 \%$ & $21.7 \%$ \\
\hline
\end{tabular}

Fuente: formularios de recolección de datos

Elaborado por las autoras 


\section{THE ECUADOR JOURNAL OF MEDICINE}

\section{I S C US I ÓN}

La automedicación es una práctica ampliamente extendida, por lo tanto, es un problema de salud pública y es más frecuente entre 15 y 34 años. Además, los medios de comunicación, especialmente el internet, han facilitado su accesibilidad (1).

En el 2011 en estudiantes venezolanos (240 estudiantes de la Universidad Pedagógica Experimental Libertador de Caracas) se observó una práctica de automedicación irresponsable en el $87 \%$ a pesar que reconocen las implicaciones de efectos adversos (1). En contraste con la población de estudio que el $72,9 \%$ se automedica con drogas como ansiolíticos, estimulantes, antidepresivos, relajantes musculares, anfetaminas o cocaína $(\mathrm{o}<0,001)$.

Se observó consumo de las drogas antes mencionadas en el $78,1 \%$ en medicina y $68 \%$ en otras facultades (p 0,015), datos que se aproximan a los de la Universidad Técnica del Norte de lbarra en estudiantes de enfermería en donde el $88 \%$ se automedican, evidenciando mayor frecuencia de consumo en carreras relacionadas con ciencias de la salud (11).

Los datos más cercanos a nuestra realidad son los recabados por la Universidad San Francisco de Quito $(n=174)$, en la escuela de medicina, en donde la prevalencia de automedicación fue 68,4\%. En el grupo de medicina el 78,1\% afirmó automedicarse con alguno de los fármacos de estudio (p $<0,024)$ (12). Llama la atención el elevado porcentaje de automedicación en la población descrita, pese al alto grado de conocimiento que implica su carrera.

En la facultad de medicina de la Universidad del Azuay el 96,4\% consumen algún tipo de sustancia estimulante. En comparación con un estudio realizado en el 2007 en la Universidad de Valparaíso, Chile, Francisco Asencio tomó una muestra de 120 estudiantes de medicina, 92,5\% consumían algún tipo de sustancia para aumentar su rendimiento las más frecuentes fueron café (80\%) seguido de modafinilo, cafeína y anfetamina ( $p$ $0,100)(9)$. Esto pudiera tener relación tanto con la exigencia académica que aumenta progresivamente, como la accesibilidad para adquirirlas. En contraste con otras facultades en donde el porcentaje fue aún mayor (98\%), probablemente atribuido al desconocimiento de sus efectos adversos.

La diferencia no fue significativa entre los grupos de estudio (p 0,278). A pesar, que el porcentaje de automedicación específicamente con drogas estimulantes como modafinilo, cafeína y ritalina si lo fue (p $<0,001)$. En ambos grupos se afirmó mayor consumo de dichas sustancias durante exámenes. Los datos obtenidos son similares a los establecidos por estudios realizados en Sudamérica como en la Universidad del Nordeste de Argentina y en poblaciones universitarias en España que se aproximan al $90 \%$ de automedicación en facultades relacionadas con ciencias de la salud con el objetivo de concentrarse o aumentar el estado de alerta $(9,10)$.

En este estudio el consumo de bebidas estimulantes fue elevado en ambos grupos entre 92-94\%, sin embargo, no mostró significancia estadística (p 0.380). El consumo de bebidas energizantes predomina en momentos de alta demanda de trabajo, siendo este el de mayor uso en el caso de la facultad de medicina con un $84 \%$. A diferencia de las otras facultades en donde su mayor consumo fue del café con un $80 \%$.

En la Universidad de Costa Rica en el 2012 se tomó una muestra de 91 estudiantes de segundo año de medicina, identificando al $89,01 \%$ como consumidores de bebidas estimulantes, en orden de frecuencia estas fueron café, Coca-Cola, bebidas energizantes, similar a datos de éste estudio en el grupo de medicina con 92\% (p 0,382). Datos que coinciden con un estudio realizado en el 2006, basado en una población de 


\section{THE ECUADOR JOURNAL OF MEDICINE}

4271 estudiantes universitarios, afirmando el $94 \%$ de las personas, haber consumido bebidas energéticas mezcladas con alcohol en los últimos 30 días $(13,14)$. Al igual que valores encontrados en una investigación liderada por médicos egresados de la Escuela de Medicina San Juan Bautista (Puerto Rico), en 296 estudiantes, el $87 \%$ de primer año de medicina consumieron en exceso estimulantes tales como cafeína (56.2\%), bebidas energizantes (27\%), anfetaminas (8\%), nicotina (6.9\%) y otros (1.8\%); comparados con la facultad de medicina (p 0,0504) (15).
Tanto en esta investigación como en otras, se evidencia que los estudiantes utilizan estas bebidas para evitar sentirse cansados, tratar la deprivación de sueño y mejorar su desempeño.

La mayoría de los estudiantes alrededor de dos tercios de la población estudiada presentaron efectos adversos (80\% medicina, $73 \%$ otras facultades, p 0,027) entre los más comunes neurológicos y cardiológicos. En ciertos países europeos como Noruega, Francia, Dinamarca su venta es prohibida, debido al alto riesgo que dicha conducta representa (16).

\section{CONCLUS I ON}

La automedicación y el consumo de bebidas estimulantes en estudiantes de la universidad del Azuay son similares a otros estudios. Los estudiantes de medicina consumen más estimulantes que las otras facultades.

\section{RECOMENDAC I ONES}

Dar a conocer a las autoridades de la institución para que tomen medidas acerca de este problema.

Educar a la población con charlas informativas sobre las bebidas y medicamentos estimulantes y los efectos que estos producen en el cuerpo.

Solicitar al departamento de psicología que colabore con asesoría a quien consu ma este tipo de sustancias sin receta médica.

\section{AGRADECIMIENTOS}

Queremos expresar nuestro sincero agradecimiento a nuestros padres, tutor y asesor metodológico quienes han formado parte en la elaboración de este proyecto. 


\section{THE ECUADOR JOURNAL OF MEDICINE}

\section{CONTRIBUCIONES DE AUTORIA}

Todos los autores han realizado a partes iguales la redacción y

argumentación del estudio.

\section{CONFLICTO DE INTERES}

Los autores declaran que no hay conflictos de interés.

\section{CORRESPONDENCIA}

Email: nataliapacurucu@gmail.com

Email: editor@revistafecim.org

\section{ITAS B I BLIOGRÁFICAS}

1. Kregar G, Filinger E. ¿Qué se entiende por automedicación?. Latin American journal of pharmacy[Internet]2005[consultado febrero 2013];24(1):p130133. Disponible en: http://www.latamjpharm.org/ trabajos/24/1/LAJOP_24_1_6_2_5ROG2AU4L2.pdf 2. Aràoz N, Aguirre J. Automedicación en el internado rotatorio. Revista de posgrado de la VIa cátedra de medicina[Internet]2009[consultado febrero 2013];1(1):1-18. Disponible en: https://med.unne. edu.ar/sitio/multimedia/imagenes/ckfinder/files/files/revista/posgrado\%20medicina\%20VI/197.pdf

3. Castronuovo C, chiclana F. Automedicación en Estudiantes de la Universidad Nacional de la Plata Argentina. Latin American Journal of Pharmacy. 2007; 26 (6), p937-944.

4. Guillem P, Bozal F. Estudio sobre Automedicación en Población Universitaria Española. Revista clinica de medicina familiar.2010; 3 (2), p99-103.

5. Vera O, Rodas C. La Automedicación: una problemática nacional y regional que se debe prevenir.
Revista del Cuerpo Médico del Hospital Nacional Almanzor Aguinaga Asenjo.201; 4 (2), p129-131.

6. Vera O. Diseño y Validación de un Cuestionario para medir Automedicación (CAuM-ovr) en estudiantes Universitarios. Revista del cuerpo médico. 2013; 6(1) p19-24.

7. De Pablo M. La automedicación en la cultura universitaria. Revista de Investigación. 2011;73(35), p219-239.

8. Asencio F, Cardemil F. Prevalancia y factores asociados al uso de drogas, fármacos y otras sustancias por estudiantes de medicina para mejorar el rendimiento académico. CIMEL. 2009, 14 (2), p87-92.

9. Grela C, Alvarez S. AUTOMEDICACION: Experiencia en estudiantes de $5^{\circ}$ año de la Facultad de Medicina de la Universidad Nacional Del Nordeste. Revista de Posgrado de la vía Cátedra de Medicina.2006; 155, p5-8.

10. Junges J. Consumo de estimulantes en estudiantes de medicina[Internet]. Buenos Aires: 2005. [con- 


\section{THE ECUADOR JOURNAL OF MEDICINE}

sultado enero 2013]. Disponible en: http://imgbiblio. vaneduc.edu.ar/fulltext/files/TC087507.pdf 2005 11. Morillo M, Vanegas V. Factores relacionados con la automedicación en los estudiantes de enfermería de la facultad ciencias de la salud de la universidad Técnica del Norte, en la ciudad de Ibarra en el periodo enero- agosto 2010[Internet] Ibarra: 2011[consultado Febrero 2013], Disponible en:http://repositorio.utn.edu.ec/bitstream/123456789/696/1/06\%20 ENF\%20427\%20ART\%C3\%8DCULO\%20CIENT\%C3\%8DFICO.pdf

12. Almeida M. Prevalencia y Prácticas de Automedicación en los estudiantes de Primero a Quinto año de la Facultad de Medicina de la Universidad de San Francisco de Quito en Octubre del 2009[Internet] Quito: 2009 [consultado Febrero 2013], Disponible en: https://repositorio.usfq.edu.ec/handle/23000/763

13. Arguedas G, Garnier M. Aspectos médico-legales de los patrones de consumo de bebidas energéticas por parte de los estudiantes de medicina de segundo año de la universidad de costa rica. Revista medicina legal de Costa Rica. 2012;29(1),p23-33.

14. Kapner, D. Ephedra and Energy Drinks on College Campuses. Higher Education Center for Alcohol and Other Drug Abuse and Violence Prevention [Internet].EEUU: 2008 [consultado Febrero 2013], Disponible en: https://files.eric.ed.gov/fulltext/ ED537639.pdf
15. Burgos B. Alto el consumo de estimulantes entre estudiantes de medicina. Revista Puertorriqueña de medicina y salud pública [Internet]. Puerto Rico: 2008 [consultado Febrero 2013], Disponible en: http://www.medicinaysaludpublica.com/ alto-el-consumo-de-estimulantes-entre-estudiantes-de-medicina/

16. Catellanos R, Frazer G. Efectos fisiológicos de las bebidas energizantes. Revista Facultad de Ciencias Médicas. 2006; 3(1), p43-49.

17. Banerjee I, Bhadury T. Self-medication practice among undergraduate medical students in a tertiary care medical college, West Bengal. J Postgrad Med. $2012 ; 58(2): p 127-31$.

18. World Health Organization. European regional meeting on implementation of the WHO Framework Convention on Tobacco Control. Budapest, Hungary[Internet].: WHO; Budapest: 2014. [consultado febrero 2013], Disponible en: http://www.who.int/ fctc/implementation/workshops/summary_of_discussions_with_comments_from_the_parties.pd$\mathrm{f}$ ?ua $=$

ED537639.pdf
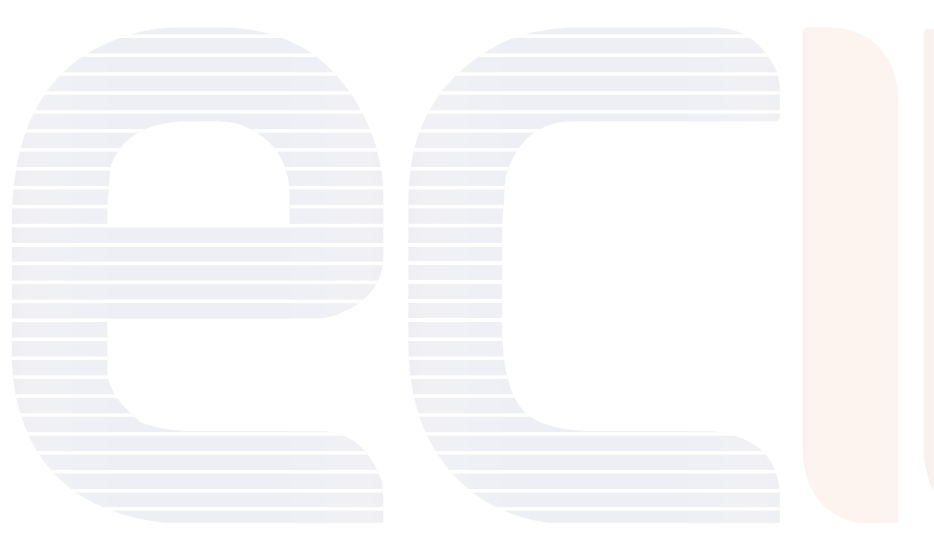